\title{
THE ASSOCIATED PRIMES OF INITIAL IDEALS OF LATTICE IDEALS
}

\author{
Serkan Hoşten and Rekha R. Thomas
}

\begin{abstract}
This paper concerns the associated primes and primary decompositions of the monomial initial ideals of lattice ideals. For a fixed initial ideal, we show that the multiplicities of its associated primes and its arithmetic degree are the cardinalities of sets of polytopes in which the origin is the unique lattice point. The associated primes are shown to exhibit a rare connectivity property: each embedded prime contains an associated prime of one higher dimension. The length of any such chain of associated primes can be bounded above by a function that depends only on the codimension of the lattice ideal. We express the unique irredundant irreducible decomposition of an initial ideal of a lattice ideal using maximal lattice point free polytopes defined by the lattice and the cost vector inducing the initial ideal.
\end{abstract}

\section{Introduction and main results}

Given a sublattice $\mathcal{L}$ of $Z^{n}$ of rank $m$, the lattice ideal of $\mathcal{L}$ is the $(n-m)$ dimensional binomial ideal

$$
I_{\mathcal{L}}:=\left\langle x^{u}-x^{v}: u-v \in \mathcal{L}\right\rangle \subseteq k\left[x_{1}, \ldots, x_{n}\right]=: k[x] .
$$

If $\mathcal{L}$ is saturated, i.e., $\left(\mathcal{L} \otimes_{Z} Q\right) \cap Z^{n}=\mathcal{L}$ then $I_{\mathcal{L}}$ is prime and there exists a matrix $A \in Z^{(n-m) \times n}$ of rank $n-m$ such that $\mathcal{L}=\left\{u \in Z^{n}: A u=0\right\}$. In this case, $I_{\mathcal{L}}$ is the toric ideal of $A$ [Ful], $[\mathrm{Stu}]$ which is denoted as $I_{A}$. Let $N$ denote the set of non-negative integers. Throughout this paper we assume that $\mathcal{L} \cap N^{n}=\{0\}$ which implies that $I_{\mathcal{L}}$ is homogeneous with respect to a degree grading where degree $\left(x_{i}\right)=a_{i}>0$. For simplicity we assume that $a_{i}=1$ for all $i=1, \ldots, n$.

In this paper we study the associated primes and primary decompositions of $\operatorname{in}_{c}\left(I_{\mathcal{L}}\right)$, the initial ideal of $I_{\mathcal{L}}$ with respect to a cost vector $c \in R^{n}$. Here $\operatorname{in}_{c}\left(I_{\mathcal{L}}\right)$ is the ideal generated by the initial forms of the polynomials in $I_{\mathcal{L}}$, with respect to $c$. In general $\operatorname{in}_{c}\left(I_{\mathcal{L}}\right)$ may not be a monomial ideal, but throughout this paper we assume that it is a monomial ideal, in which case $c$ is said to be generic. In other words, $\operatorname{in}_{c}\left(I_{\mathcal{L}}\right)=\operatorname{in}_{\succ}\left(I_{\mathcal{L}}\right)$ for some term order $\succ$, and $c$ is a vector that lies in the interior of the Gröbner cone corresponding to $\operatorname{in}_{\succ}\left(I_{\mathcal{L}}\right)$ (see [Stu], Chapters 1 and 2). Since $\mathcal{L} \cap N^{n}=\{0\}$, every Gröbner cone of $I_{\mathcal{L}}$ has a non-trivial intersection with the non-negative orthant of $R^{n}$, and hence we may assume that $c$ is a non-zero non-negative integral vector. In Section 3 we show

Received August 28,1998. 
that the associated primes of $\operatorname{in}_{c}\left(I_{\mathcal{L}}\right)$ come in saturated chains which is a rare property for an arbitrary monomial ideal.

Theorem 3.1 If $P$ is an embedded prime of $i n_{c}\left(I_{\mathcal{L}}\right)$, then $P$ contains an associated prime $Q$ of $i n_{c}\left(I_{\mathcal{L}}\right)$ such that $\operatorname{dim}(Q)=\operatorname{dim}(P)+1$.

Let $\operatorname{Ass}\left(\operatorname{in}_{c}\left(I_{\mathcal{L}}\right)\right)$ denote the partially ordered set (poset) of associated primes of $\operatorname{in}_{c}\left(I_{\mathcal{L}}\right)$ where $Q \geq P$ if $Q \subseteq P$. The minimal primes of $\operatorname{in}_{c}\left(I_{\mathcal{L}}\right)$ are hence the maximal elements of $\operatorname{Ass}\left(\operatorname{in}_{c}\left(I_{\mathcal{L}}\right)\right)$. In Section 3 we also show that the length of a maximal chain in $\operatorname{Ass}\left(\operatorname{in}_{c}\left(I_{\mathcal{L}}\right)\right)$ can be bounded above by a function that depends only on the codimension of $I_{\mathcal{L}}$ and that this bound is sharp. This result also follows from Theorem 2.3 in [PS].

Theorem 3.5 The length of a maximal chain in $A s s\left(i n_{c}\left(I_{\mathcal{L}}\right)\right)$ is at most min $(n-$ $\left.m, 2^{m}-(m+1)\right)$.

The associated primes of a monomial ideal $M$ are closely related to the standard pair decomposition [STV] of the standard monomials of $M$ which we now describe. Recall that a monomial $x^{m}$ is a standard monomial of $M$ if $x^{m} \notin M$. For $m \in N^{n}$, let the support of $m$ be denoted by $\operatorname{supp}(m):=\left\{i: m_{i} \neq 0\right\}$ and let $\operatorname{supp}\left(x^{m}\right):=\operatorname{supp}(m)$. Let $[n]:=\{1, \ldots, n\}$.

Definition 1.1. For a monomial $x^{m} \in k[x]$ and $\sigma \subseteq[n]$, we say that $\left(x^{m}, \sigma\right)$ is an admissible pair of a monomial ideal $M$ if: (i) $\operatorname{supp}(m) \cap \sigma=\emptyset$, and (ii) every monomial in $x^{m} \cdot k\left[x_{j}: j \in \sigma\right]$ is a standard monomial of $M$.

There is a natural partial order on the set of all admissible pairs of $M$ given by $\left(x^{m}, \sigma\right) \leq\left(x^{m^{\prime}}, \sigma^{\prime}\right)$ if and only if $x^{m}$ divides $x^{m^{\prime}}$ and $\operatorname{supp}\left(x^{m^{\prime}} / x^{m}\right) \cup \sigma^{\prime} \subseteq \sigma$.

Definition 1.2. An admissible pair $\left(x^{m}, \sigma\right)$ of $M$ is called a standard pair of $M$ if it is a minimal element in the poset of all admissible pairs with respect to the above partial order.

The standard pairs of $M$ induce a unique covering of the set of standard monomials of $M$ which we refer to as the standard pair decomposition of $M$. It was shown in $[\mathrm{STV}]$ that the arithmetic degree $[\mathrm{BM}]$ of $M$, denoted arithdeg $(M)$, equals the number of standard pairs of $M$. This paper gives a bound for arithdeg $(M)$ in terms of the degrees of the minimal generators and dimension of $M$. Algebraically, $\operatorname{arithdeg}(M)=\sum \operatorname{mult}(P) \operatorname{deg}(P)$ where the sum is over all homogeneous prime ideals $P$ in $k[x]$, and $\operatorname{mult}(P)$ denotes the length of the largest ideal of finite length in the ring $k[x]_{P} / M k[x]_{P}$, and $\operatorname{deg}(P)$ is the geometric degree of the variety of $P$. The integer $\operatorname{mult}(P)$ is positive if and only if $P$ is an associated prime of $M$. In our situation, since $M$ is a monomial ideal, every associated prime of $M$ has the form $p_{\sigma}:=\left\langle x_{j}: j \notin \sigma\right\rangle$ for some $\sigma \subseteq[n]$ and, $\operatorname{deg}\left(p_{\sigma}\right)=1$ since $\operatorname{deg}\left(x_{i}\right)=1$.

Theorem 1.3. [STV] Given a monomial ideal $M$ and $p_{\sigma}=\left\langle x_{j}: j \notin \sigma\right\rangle$ for some $\sigma \subseteq[n]$ : 
(i) $p_{\sigma}$ is an associated prime of $M$ if and only if $M$ has a standard pair of the form $(\cdot, \sigma)$. If $p_{\sigma}$ is an associated prime of $M$ then mult $\left(p_{\sigma}\right)$ equals the number of standard pairs of $M$ of the form $(\cdot, \sigma)$. Hence, arithdeg $(M)=\sum_{\sigma \subseteq[n]}$ mult $\left(p_{\sigma}\right)$ is the number of standard pairs of $M$.

(ii) $p_{\sigma}$ is a minimal prime of $M$ if and only if $(1, \sigma)$ is a standard pair of $M$.

Let $B \in Z^{n \times m}$ be a matrix whose columns form a basis for $\mathcal{L}$. By our earlier assumptions, $\operatorname{ker}\left(B^{T}\right)$ contains a strictly positive vector and hence the rows of $B$ denoted as $b_{1}, \ldots, b_{n}$ span $R^{m}$ positively, i.e., cone $\left(b_{1}, \ldots, b_{n}\right)=R^{m}$. Given a generic cost vector $c \in R^{n}$, the vector $c B$ lies in the relative interior of a collection $\mathcal{C}$ of $m$-dimensional simplicial cones whose generators are in $\left\{b_{1}, \ldots, b_{n}\right\}$. We identify each such cone with the set of row indices of its generators. Then it can be shown that the complements of (the indices of the generators of) the cones in $\mathcal{C}$ form the maximal faces of an $(n-m)$-dimensional pure simplicial complex on $[n]$ (see [BFS]). For a fixed lattice $\mathcal{L}$ this simplicial complex depends only on $c$, and hence we will denote it by $\Delta_{c}$. In other words, $\tau$ is a face of $\Delta_{c}$ if and only if $P_{v}^{\bar{\tau}}(0):=\left\{u \in R^{m}:-(c B) \cdot u \leq 0, B^{\bar{\tau}} u \leq v\right\}$ is a polytope (i.e., bounded polyhedron) for all $v \in N^{|\bar{\tau}|}$ where $\bar{\tau}=[n] \backslash \tau$ and $B^{\bar{\tau}}$ is the submatrix of $B$ whose rows are indexed by $\bar{\tau}$. In Section 2 we characterize the standard monomials and standard pairs of $\operatorname{in}_{c}\left(I_{\mathcal{L}}\right)$ in terms of polytopes defined by $\mathcal{L}$ and $c$ in which the origin is the unique lattice point. These characterizations are then used to prove the results of Section 3. An important feature of our results is that the ideal $\operatorname{in}_{c}\left(I_{\mathcal{L}}\right)$ is never needed in explicit form (in terms of a minimal generating set for example) in order to obtain its associated primes, irreducible decomposition and standard pairs. The two main results of Section 2 are as follows:

Theorem 2.3 The monomial $x^{v}$ is a standard monomial of $i n_{c}\left(I_{\mathcal{L}}\right)$ if and only if 0 is the unique lattice point in $P_{v}(0):=\left\{u \in R^{m}: B u \leq v,-(c B) \cdot u \leq 0\right\}$.

Theorem 2.5 An admissible pair $\left(x^{v}, \tau\right)$ of $i n_{c}\left(I_{\mathcal{L}}\right)$ is a standard pair if and only if 0 is the unique lattice point in $P_{v}^{\bar{\tau}}(0)$ and all of the inequalities in the system $B^{\bar{\tau}} u \leq v$ are essential, i.e., removing any inequality introduces a new lattice point into the resulting polyhedron.

Theorems 2.3 and 2.5 give the following combinatorial interpretation of the mutliplicity of an associated prime of $\operatorname{in}_{c}\left(I_{\mathcal{L}}\right)$ and the arithmetic degree of $\operatorname{in}_{c}\left(I_{\mathcal{L}}\right)$

\section{Corollary 2.7}

(i) The multiplicity of $p_{\tau}$ is the number of polytopes of the form $P_{v}^{\bar{\tau}}(0)$ where $v \in N^{|\bar{\tau}|}, 0$ is the unique lattice point in $P_{v}^{\bar{\tau}}(0)$ and all inequalities in $B^{\bar{\tau}} u \leq v$ are essential. 
(ii) The arithmetic degree of $i_{c}\left(I_{\mathcal{L}}\right)$ is the total number of such polytopes $P_{v}^{\bar{\tau}}(0)$ as $\tau$ ranges over the subsets of $[n]$.

We conclude in Section 4 with an explicit formula for an irreducible primary decomposition of $\operatorname{in}_{c}\left(I_{\mathcal{L}}\right)$ in terms of the maximal lattice point free polytopes [BSS], [Lov] of the form $P_{v}^{\bar{\tau}}(0)$. The crucial idea here is to prove a bijection between the socle elements in the localization of $\operatorname{in}_{c}\left(I_{\mathcal{L}}\right)$ at the associated prime $p_{\tau}$ and the maximal lattice point free polytopes of the form $P_{v}^{\bar{\tau}}(0)$.

\section{Multiplicity of associated primes and arithmetic degree}

Two lattice points $v, u \in N^{n}$ are congruent modulo $\mathcal{L}$ if $v-u \in \mathcal{L}$. The congruence classes of $N^{n}$ modulo $\mathcal{L}$ are called the fibers of $\mathcal{L}$. Since $\mathcal{L} \cap N^{n}=\{0\}$, each fiber $C \in N^{n} / \mathcal{L}$ is finite, and since the cost vector $c$ is generic, each fiber contains a unique lattice point that minimizes the linear functional $c \cdot x$ (follows from Section 5 in [SWZ]). These optimal solutions define $\operatorname{in}_{c}\left(I_{\mathcal{L}}\right)$.

Proposition 2.1. [SWZ] A monomial $x^{v}$ is a standard monomial of $i n_{c}\left(I_{\mathcal{L}}\right)$ if and only if $v$ minimizes the linear functional $c \cdot x$ in its fiber.

Given a fiber $C$ of $\mathcal{L}$ and $v \in C$ we can identify this fiber with the lattice points in the polytope

$$
P_{v}:=\left\{u \in R^{m}: B u \leq v\right\},
$$

via the map $P_{v} \cap Z^{m} \rightarrow N^{n}$ such that $u \rightarrow v-B u$. In particular, $0 \in P_{v}$ maps to $v \in C$. If $v, v^{\prime} \in C$, then $P_{v}$ and $P_{v^{\prime}}$ are lattice translates of each other and hence we may represent $C$ by any of the polytopes $P_{v}$ where $v \in C$. This representation of a fiber of $\mathcal{L}$ was introduced in [PS].

Proposition 2.2. Let $v$ be an element of a fiber $C$. Then the monomial $x^{\left(v-B u^{*}\right)}$ is a standard monomial of $i n_{c}\left(I_{\mathcal{L}}\right)$ if and only if $u^{*}$ is the optimal solution to the integer program

$$
\text { minimize }\left\{-(c B) \cdot u: u \in P_{v} \cap Z^{m}\right\} \text {. }
$$

Proof. By Proposition 2.1, the monomial $x^{v^{*}}$ is a standard monomial of $\operatorname{in}_{c}\left(I_{\mathcal{L}}\right)$ if and only if the lattice point $v^{*} \in C$ minimizes $c \cdot x$ over $C$. This happens:

$\Longleftrightarrow$ there exists $u^{*} \in Z^{m}$ such that $v^{*}=v-B u^{*} \geq 0$ and $c\left(v-B u^{*}\right)<$ $c(v-B u)$ for all $u \neq u^{*} \in Z^{m}$ with $v-B u \geq 0$

$\Longleftrightarrow$ there exist $u^{*} \in P_{v} \cap Z^{m}$ such that $-(c B) \cdot u^{*}<-(c B) \cdot u$ for all $u \in P_{v} \cap Z^{m}$ and $u \neq u^{*}$

$\Longleftrightarrow u^{*}$ is the optimal solution of the integer program (2).

Using the optimal solution $u^{*}$ to the program (2), we define the following subpolytope of $P_{v}$ which plays an important role in this paper:

$$
P_{v}\left(u^{*}\right):=\left\{u \in R^{m}: B u \leq v,-(c B) \cdot u \leq-(c B) \cdot u^{*}\right\} .
$$


Theorem 2.3. Let $v \in N^{n}$ be a lattice point in a fiber $C$. Then $x^{v-B u^{*}}$ is a standard monomial of $i n_{c}\left(I_{\mathcal{L}}\right)$ if and only if $u^{*}$ is the unique lattice point in $P_{v}\left(u^{*}\right)$. In particular, $x^{v}$ is a standard monomial of $i n_{c}\left(I_{\mathcal{L}}\right)$ if and only if 0 is the unique lattice point in $P_{v}(0)=\left\{u \in R^{m}: B u \leq v,-(c B) \cdot u \leq 0\right\}$.

Proof. Since $c$ is assumed to be generic, each integer program (2) has a unique optimal solution. By Proposition 2.2, $u^{*} \in Z^{m}$ is the optimal solution to (2) if and only if $u^{*}$ is in $P_{v}$ and there is no $u \neq u^{*}$ in $P_{v} \cap Z^{m}$ such that $-(c B) \cdot u \leq$ $-(c B) \cdot u^{*}$. This is equivalent to $u^{*}$ being the unique lattice point in $P_{v}\left(u^{*}\right)$. The second statement follows immediately.

Example 2.4. Consider the lattice $\mathcal{L}$ of rank two which is generated by the rows of

$$
B^{T}=\left[\begin{array}{rrrrr}
5 & 2 & -6 & 5 & -6 \\
-1 & 1 & -1 & 0 & 1
\end{array}\right] .
$$

The codimension two lattice ideal $I_{\mathcal{L}}$ is homogeneous with respect to the usual total degree grading. Let $c=\left(\begin{array}{lllll}6 & 2 & 1 & 1 & 7\end{array}\right)$ and consider the fiber $C$ of $v=$ $(2,0,5,2,3)$. The polytope $P_{v}=\left\{u \in R^{2}: B u \leq v\right\}$ is defined by the five inequalities whose normal vectors are the columns $b_{1}, \ldots, b_{5}$ of $B^{T}$ (see Figure 1 ). Observe that there are three lattice points in $P_{v}:(0,0),(0,-1),(0,-2)$. They correspond to the vectors $(2,0,5,2,3),(1,1,4,2,4),(0,2,3,2,5)$ in $C$, respectively. If we add the constraint $-(c B) \cdot u \leq 0$ to $P_{v}$ where $-(c B)=(9,-2)$ we get $P_{v}(0)$. Figure 1 shows that 0 is the unique lattice point in $P_{v}(0)$, and Theorem 2.3 implies that $x^{v}$ is a standard monomial of $\operatorname{in}_{c}\left(I_{\mathcal{L}}\right)$. Indeed, the reduced Gröbner basis of $I_{\mathcal{L}}$ with respect to $c$ is $\mathcal{G}_{c}=\left\{x_{3}^{4} x_{5}^{8}-x_{1}^{7} x_{4}^{5}, x_{3}^{5} x_{5}^{7}-\right.$ $x_{1}^{6} x_{2} x_{4}^{5}, x_{3}^{6} x_{5}^{6}-x_{1}^{5} x_{2}^{2} x_{4}^{5}, x_{3}^{7} x_{5}^{5}-x_{1}^{4} x_{2}^{3} x_{4}^{5}, x_{3}^{8} x_{5}^{4}-x_{1}^{3} x_{2}^{4} x_{4}^{5}, x_{3}^{9} x_{5}^{3}-x_{1}^{2} x_{2}^{5} x_{4}^{5}, x_{3}^{10} x_{5}^{2}-$ $\left.x_{1} x_{2}^{6} x_{4}^{5}, x_{2} x_{5}-x_{1} x_{3}, x_{2}^{7} x_{4}^{5}-x_{3}^{11} x_{5}\right\}$ with respect to which $x_{1}^{2} x_{3}^{5} x_{4}^{2} x_{5}^{3}$ is a standard monomial.

For $\tau \subseteq[n]$, let $P_{v}^{\bar{\tau}}:=\left\{u \in R^{m}: B^{\bar{\tau}} u \leq v^{\bar{\tau}}\right\}$ be the polyhedron obtained from $P_{v}$ by removing the inequalities indexed by $\tau$. Here we use $v^{\bar{\tau}}$ to denote the truncation of $v$ obtained by restricting to the coordinates indexed by $\bar{\tau}\left(v^{\bar{\tau}}\right.$ lies in $\left.N^{|\bar{\tau}|}\right)$. Let $P_{v}^{\bar{\tau}}(0):=\left\{u \in R^{m}:(-c B) \cdot u \leq 0, B^{\bar{\tau}} u \leq v^{\bar{\tau}}\right\}$ which is gotten from $P_{v}^{\bar{\tau}}$ by adding the additional constraint $(-c B) \cdot u \leq 0$. For a polyhedron $P=\left\{x \in R^{p}: T_{i} x \leq t_{i}, i=1, \ldots, q\right\}$ we say that an inequality $T_{i} x \leq t_{i}$ is essential if the relaxation of the polyhedron obtained by removing $T_{i} x \leq t_{i}$ contains a new lattice point.

Theorem 2.5. An admissible pair $\left(x^{v}, \tau\right)$ of $i n_{c}\left(I_{\mathcal{L}}\right)$ is a standard pair if and only if 0 is the unique lattice point in $P_{v}^{\bar{\tau}}(0)$ and all of the inequalities in the system $B^{\bar{\tau}} u \leq v^{\bar{\tau}}$ are essential.

Proof. An admissible pair $\left(x^{v}, \tau\right)$ of $M:=\operatorname{in}_{c}\left(I_{\mathcal{L}}\right)$ is a standard pair if and only if all monomials in $x^{v} \cdot k\left[x_{j}: j \in \tau\right]$ are standard monomials, and for every $i \in \bar{\tau}$ there exists $m_{i}>0$ such that $x_{i}^{m_{i}} \cdot x^{v} \in M$. By Theorem 2.3, this is the case if and only if for every $v^{\prime}$ with support in $\tau$, the origin is the unique lattice point in 

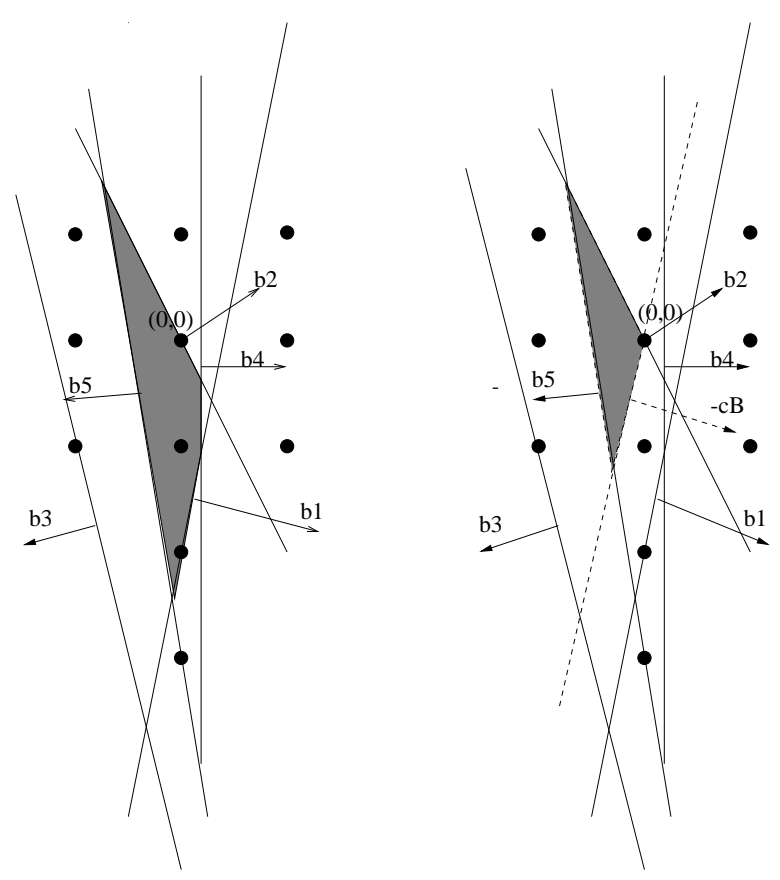

Figure $1 . P_{v}$ and $P_{v}(0)$ of Example 2.4.

$P_{\left(v+v^{\prime}\right)}(0)=\left\{u \in R^{m}: B^{\bar{\tau}} u \leq v^{\bar{\tau}}, B^{\tau} u \leq v^{\prime \tau},(-c B) \cdot u \leq 0\right\}$ and for each $i \in \bar{\tau}$ there exists $m_{i}>0$ such that $P_{\left(v_{i}+v^{\prime}\right)}(0)$ where $v_{i}=v+\left(0, \ldots, 0, m_{i}, 0, \ldots, 0\right)$ contains an extra lattice point. This is equivalent to saying that $P_{v}^{\bar{\tau}}(0)$ contains the origin as the unique lattice point, and each inequality in this polytope is essential.

Example 2.6. Example 2.4 continued. If we examine $P_{v}(0)$ in this example, we see that removing the inequalities corresponding to $\tau=\{1,3,4\}$ keeps 0 as the unique lattice point in the resulting polytope. But we cannot take a superset of $\tau$ and maintain the same property. Hence $\left(x_{5}^{3}, \tau\right)$ is a standard pair of $\operatorname{in}_{c}\left(I_{\mathcal{L}}\right)$.

Using Theorem 2.5 we obtain a combinatorial interpretation for $\operatorname{mult}\left(p_{\tau}\right)$ and $\operatorname{arithdeg}\left(\operatorname{in}_{c}\left(I_{\mathcal{L}}\right)\right)$.

\section{Corollary 2.7.}

(i) The multiplicity of $p_{\tau}$ is the number of polytopes of the form $P_{v}^{\bar{\tau}}(0):=$ $\left\{u \in R^{m}: B^{\bar{\tau}} u \leq v^{\bar{\tau}},-(c B) \cdot u \leq 0\right\}$ where $v \in N^{n}$, 0 is the unique lattice point in $P_{v}^{\bar{\tau}}(0)$ and all inequalities in $B^{\bar{\tau}} u \leq v^{\bar{\tau}}$ are essential.

(ii) The arithmetic degree of $i n_{c}\left(I_{\mathcal{L}}\right)$ is the total number of such polytopes $P_{v}^{\bar{\tau}}(0)$ as $\tau$ ranges over the subsets of $[n]$. 
In the rest of this section we will relate the faces of the simplicial complex $\Delta_{c}$ from the introduction to the associated primes of $\operatorname{in}_{c}\left(I_{\mathcal{L}}\right)$. We start with the maximal faces of $\Delta_{c}$.

Theorem 2.8. The prime ideal $p_{\sigma}$ is a minimal prime of $i_{c}\left(I_{\mathcal{L}}\right)$ if and only if $\sigma$ is a maximal face of $\Delta_{c}$. The multiplicity of such a minimal prime is $\left|\operatorname{det}\left(B^{\bar{\sigma}}\right)\right|$.

Proof. If $\sigma$ is a maximal face of $\Delta_{c}$, by the definition of this simplicial complex, the vector $c B$ is in the interior of the full-dimensional simplicial cone generated by the rows of $B^{\bar{\sigma}}$. Therefore $P_{v}^{\bar{\sigma}}(0)$ is a polytope for all $v \in N^{n}$ defined by $m+1$ inequalities $\left(P_{v}^{\bar{\sigma}}(0)\right.$ is a full dimensional simplex in $\left.R^{m}\right)$, and if we omit any one of these inequalities the resulting polyhedron will be unbounded. In particular, when $v=0$ the polytope $P_{v}^{\bar{\sigma}}(0)$ is just the origin itself and each inequality is essential. This proves that $(1, \sigma)$ is a standard pair of $\operatorname{in}_{c}\left(I_{\mathcal{L}}\right)$, and by Theorem 1.3 (ii) $p_{\sigma}$ is a minimal prime. Conversely, if $p_{\sigma}$ is a minimal prime then $(1, \sigma)$ is a standard pair. Hence $P_{0}^{\bar{\sigma}}(0)$ is bounded, and therefore there exists a strictly positive linear relation on $-(c B)$ and the rows of $B^{\bar{\sigma}}$, i.e, $s(-c B)+\sum_{i \notin \sigma} s_{i} b_{i}=0$ for some $s, s_{i}>0$. This means that the vector $c B$ is in the relative interior of the cone generated by the rows of $B^{\bar{\sigma}}$, and hence $\sigma \subseteq \tau$ for a maximal face $\tau$ of $\Delta_{c}$. By the first part of the proof, $p_{\tau}$ is a minimal prime and hence $\sigma=\tau$.

For the second claim, first note that $B^{\bar{\sigma}}$ is an $m \times m$ nonsingular matrix since its rows generate a full-dimensional simplicial cone in $R^{m}$. Since the lattice $\mathcal{L}^{\prime}$ generated by the columns of $B^{\bar{\sigma}}$ has index $\left|\operatorname{det}\left(B^{\bar{\sigma}}\right)\right|$ in $Z^{m}$, and since such a lattice will always contain a strictly positive vector, the number of fibers of $N^{m} / \mathcal{L}^{\prime}$ is equal to this index. Hence for each such fiber there exists $v \in N^{n}$ with support in $\bar{\sigma}$ such that $P_{v}^{\bar{\sigma}}(0)$ contains the origin as the unique lattice point. Since omitting any inequality defining this polytope gives rise to an unbounded set, we conclude that $\left(x^{v}, \sigma\right)$ is a standard pair. Using Corollary 2.7 (i) we see that $\left|\operatorname{det}\left(B^{\bar{\sigma}}\right)\right|$ is the multiplicity of $p_{\sigma}$.

Corollary 2.9. The radical of $i n_{c}\left(I_{\mathcal{L}}\right)$ is the Stanley-Reisner ideal of the simplicial complex $\Delta_{c}$. Moreover, if $p_{\tau}$ is an associated prime of $i n_{c}\left(I_{\mathcal{L}}\right)$, then $\tau$ is a face of $\Delta_{c}$.

Proof. The Stanley-Reisner ideal of a finite simplicial complex is the ideal generated by all monomials $x_{i_{1}} \cdots x_{i_{k}}$ such that $\left\{i_{1}, \ldots, i_{k}\right\}$ is a non-face of the complex. By Theorem 2.8 the radical of $\operatorname{in}_{c}\left(I_{\mathcal{L}}\right)$ is the intersection of $p_{\sigma}$ as $\sigma$ runs through the maximal faces of $\Delta_{c}$.

The above corollary also shows the well known fact that $\operatorname{in}_{c}\left(I_{\mathcal{L}}\right)$ is an equidimensional ideal (all minimal primes have the same dimension $n-m$ ). This follows from the fact that $\Delta_{c}$ is a pure simplicial complex whose maximal faces have dimension $n-m$.

When the lattice ideal $I_{\mathcal{L}}$ is the toric ideal $I_{A}$, the simplicial complex $\Delta_{c}$ is the regular triangulation of $A$ induced by $c$, and Theorem 2.8 and Corollary 2.9 for the toric case can be found in Chapter 8 of [Stu]. To illustrate Theorem 


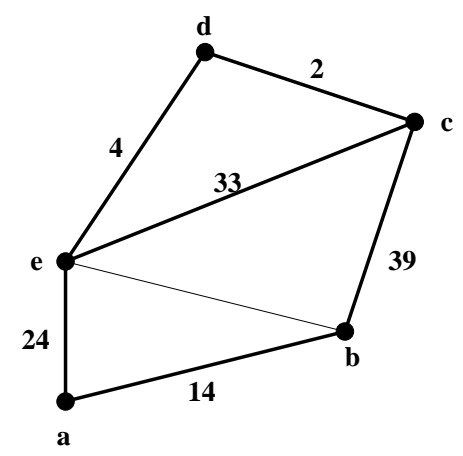

Figure 2. The bold faces of $\Delta_{c}$ index the embedded primes of $\operatorname{in}_{c}\left(I_{\mathcal{L}}\right)$.

2.5 and Theorem 2.8 we give the following example in which the lattice ideal is toric.

Example 2.10. Consider

$$
A=\left[\begin{array}{lllll}
1 & 1 & 1 & 1 & 1 \\
0 & 4 & 5 & 2 & 0 \\
0 & 1 & 4 & 5 & 2
\end{array}\right]
$$

and $c=(17,21,0,0,0)$. We let $a, b, z, d, e$ be the variables indexing the five columns of $A$. Then $\mathcal{L}=\operatorname{ker}(A) \cap Z^{5}$ is generated by the rows of

$$
B^{T}=\left[\begin{array}{ccccc}
-8 & 7 & -6 & 1 & 6 \\
-13 & 10 & -8 & 0 & 11
\end{array}\right]
$$

and $-(c B)=(-11,11)$. Since the columns of $A$ lie on the hyperplane $x_{1}=1$, we can draw (the regular triangulation) $\Delta_{c}$ as a triangulation of a pentagon as in Figure 2. Recall that $\Delta_{c}$ is a triangulation of the cone in $R^{3}$ generated by the five columns of $A$, and the pentagon in Figure 2 is the intersection of this cone with the hyperplane $\left\{x \in R^{3}: x_{1}=1\right\}$. The minimal primes of $\operatorname{in}_{c}\left(I_{\mathcal{L}}\right)$ are in bijection with the three maximal faces of $\Delta_{c}$. The simplices $\tau \in \Delta_{c}$ such that $p_{\tau}$ is an embedded prime of $\operatorname{in}_{c}\left(I_{\mathcal{L}}\right)$ are the faces of $\Delta_{c}$ indicated by bold lines. The multiplicity of each embedded prime is given next to the corresponding face. In particular, all embedded primes of $\operatorname{in}_{c}\left(I_{\mathcal{L}}\right)$ have dimension two and $p_{\{2,5\}}$ is not an embedded prime.

Consider $\tau=\{3,5\}$ in $\Delta_{c}$. We group the monomials appearing in the 33 standard pairs of the form $(\cdot,\{3,5\})$ into two groups depending on the exponent of the variable $d$ :

Group 1: (exponent of $d$ is one) $a^{2} b d, a^{2} b^{2} d, a^{2} b^{3} d, a^{2} b^{4} d, a^{2} b^{5} d, a^{2} b^{6} d, a^{3} b d$, $a^{3} b^{2} d, a^{3} b^{3} d, a^{3} b^{4} d, a^{3} b^{5} d, a^{3} b^{6} d, a^{4} b d, a^{4} b^{2} d, a^{4} b^{3} d, a^{4} b^{4} d, a^{4} b^{5} d, a^{4} b^{6} d, b^{4} d, a b^{4} d$, $b^{5} d, a b^{5} d, b^{6} d, a b^{6} d, a^{4} b^{6} d$.

Group 2:(exponent of $d$ is two) $a^{2} b d^{2}, a^{3} b d^{2}, a^{4} b d^{2}, a^{2} b^{2} d^{2}, a^{3} b^{2} d^{2}, a^{4} b^{2} d^{2}$, $a^{2} b^{3} d^{2}, a^{3} b^{3} d^{2}, a^{4} b^{3} d^{2}$. 


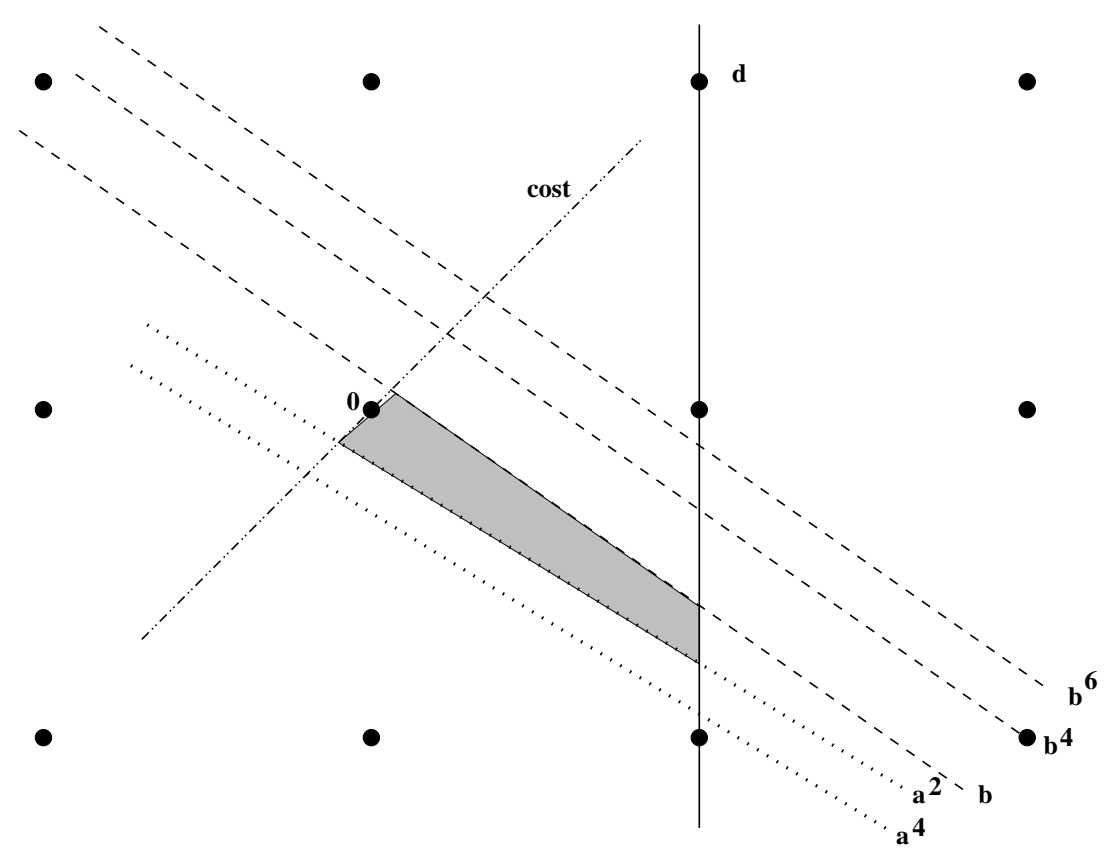

Figure 3. The polytopes $P_{v}^{\bar{\tau}}(0)$ for the monomials in Group 1.

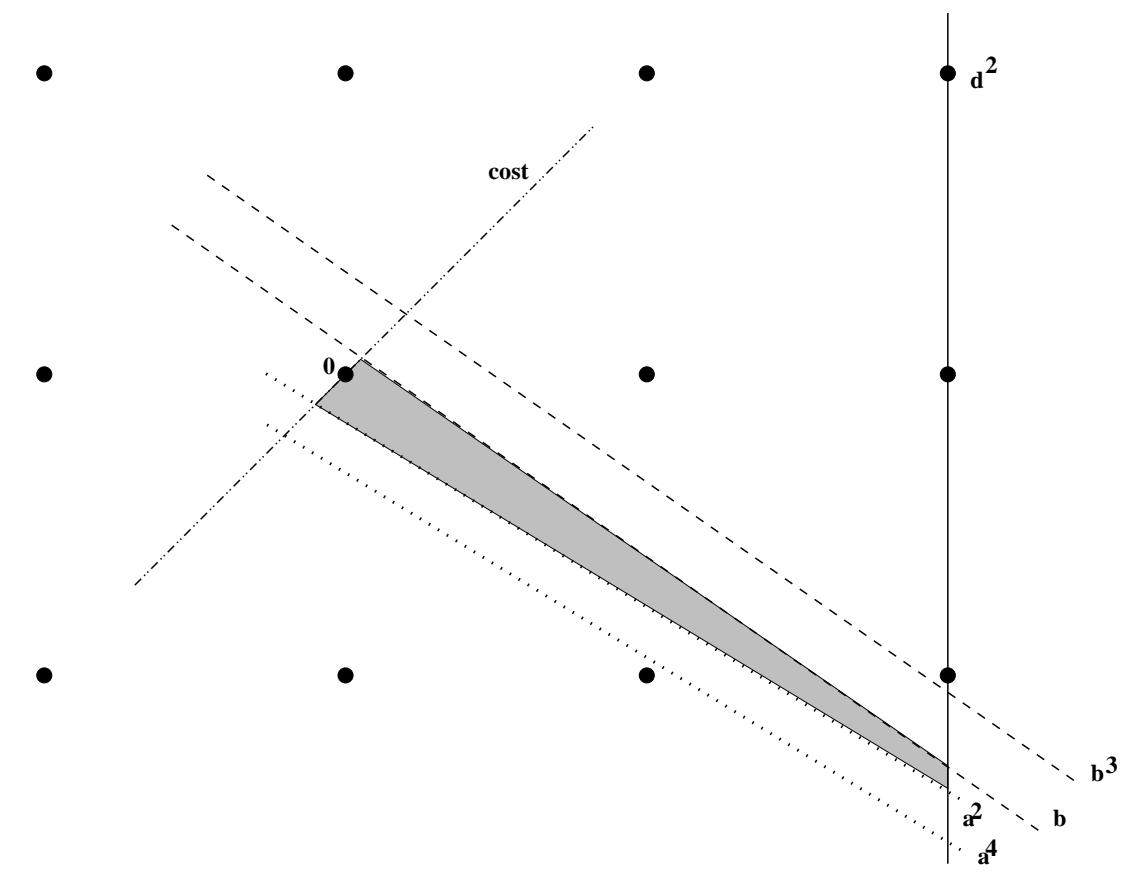

Figure 4. The polytopes $P_{v}^{\bar{\tau}}(0)$ for the monomials in Group 2. 
We first consider Group 1. The shaded polytope in Figure 3 is $P_{v}^{\bar{\tau}}(0)$ for the monomial $a^{2} b d$. Each inequality is labeled by a monomial in the variable indexing the inequality. The exponent of this monomial is the component of $v$ in the slot indexed by this variable. In Figure 3, the inequality indexed by $d$ is fixed at right hand side value one. Notice that 0 is the unique lattice point in the shaded polytope and that all the three inequalities (indexed by $a, b, d$ ) are essential for the shaded polytope (if you remove inequality $d$, the lattice point $(3,-2)$ enters the relaxation). By Theorem 2.5, Figure 3 can be used to compute all the monomials in Group 1 by translating inequalities $a$ and $b$ to all positions that will keep $P_{v}^{\bar{\tau}}(0)$ lattice point free except for the origin and all inequalities essential. For instance, for any right hand side value of inequality $b$ between one and six and any right hand side value of inequality $a$ between two and four $P_{v}^{\bar{\tau}}(0)$ has the properties of Theorem 2.5. This shows that the first 18 monomials in Group 1 appear in standard pairs with $\tau=\{3,5\}$. At the right hand side values between four and six for inequality $b$, you can decrease the right hand side of inequality $a$ from two to zero while still maintaining the required properties for $P_{v}^{\bar{\tau}}(0)$. This takes care of the last six monomials in Group 1. Figure 4 illustrates Theorem 2.5 for the monomials in Group 2.

\section{The poset of associated primes}

We now establish a structural result for $\operatorname{Ass}\left(\operatorname{in}_{c}\left(I_{\mathcal{L}}\right)\right)$, the poset of associated primes of $\operatorname{in}_{c}\left(I_{\mathcal{L}}\right)$ where for $P, Q \in \operatorname{Ass}\left(\operatorname{in}_{c}\left(I_{\mathcal{L}}\right)\right)$, we write $P \leq Q$ if $P \supseteq Q$. Corollary 2.9 implies that $\operatorname{Ass}\left(\operatorname{in}_{c}\left(I_{\mathcal{L}}\right)\right)$ is a subposet of the face lattice of $\Delta_{c}$, both posets having the same maximal elements.

Theorem 3.1. Let $\tau$ be a non-maximal face of $\Delta_{c}$ such that $p_{\tau}$ is an embedded prime of $i n_{c}\left(I_{\mathcal{L}}\right)$. Then there exists a $\tau^{\prime} \in \Delta_{c}$ such that $\tau^{\prime} \supset \tau,\left|\tau^{\prime}\right|=|\tau|+1$ and $p_{\tau^{\prime}}$ is an associated prime of $i n_{c}\left(I_{\mathcal{L}}\right)$.

Proof. Since $p_{\tau}$ is an embedded prime, $\operatorname{in}_{c}\left(I_{\mathcal{L}}\right)$ has a standard pair of the form $\left(x^{v}, \tau\right)$ where $v \neq 0$ and $\operatorname{supp}(v) \subseteq \bar{\tau}$. By Theorem 2.5, the origin is the unique lattice point in $P_{v}^{\bar{\tau}}(0)=\left\{u \in R^{m}: B^{\bar{\tau}} u \leq v^{\bar{\tau}},-(c B) \cdot u \leq 0\right\}$ and all the inequalities of $B^{\bar{\tau}} u \leq v^{\bar{\tau}}$ are essential. For each $i \in \bar{\tau}$, let $R^{i}$ be the relaxation of $P_{v}^{\bar{\tau}}(0)$ obtained by removing the $i$ th inequality $b_{i} \cdot u \leq v_{i}$, i.e., $R^{i}:=\left\{u \in R^{m}: B^{\overline{\tau \cup\{i\}}} u \leq v^{\overline{\tau \cup\{i\}}},-(c B) \cdot u \leq 0\right\}$. Let $E^{i}:=R^{i} \backslash P_{v}^{\bar{\tau}}(0)=\{u \in$ $\left.R^{m}: B^{\overline{\tau \cup\{i\}}} u \leq v^{\overline{\tau \cup\{i\}}}, b_{i} \cdot u>v_{i},-(c B) \cdot u \leq 0\right\}$. Clearly, $E^{i} \cap P_{v}^{\bar{\tau}}(0)=\emptyset$, and since the removal of each inequality introduces at least one lattice point into $R^{i}$ we have $E^{i} \cap Z^{m} \neq \emptyset$. Let $u_{i}^{*}$ be the optimal solution to the integer program $\min \left\{-(c B) \cdot u: u \in E^{i} \cap Z^{m}\right\}$. This integer program is always feasible since $E^{i} \cap Z^{m} \neq \emptyset$, but it may not have a finite optimal value. However, there exists at least one $i \in \bar{\tau}$ for which the above integer program is bounded. To see this, pick a maximal simplex $\sigma \in \Delta_{c}$ such that $\tau \subset \sigma$. The polytope $\left\{u \in R^{m}: B^{\bar{\sigma}} u \leq v^{\bar{\sigma}},-(c B) \cdot u \leq 0\right\}$ is a simplex and hence bounded. This polytope contains all $E^{i}$ for $i \in \sigma \backslash \tau$ and hence all these $E^{i}$ are bounded and have 
finite optima with respect to $-(c B)$. We may assume that the inequalities in $B^{\bar{\tau}} u \leq v^{\bar{\tau}}$ are labeled so that the finite optimal values are ordered as $-(c B) \cdot u_{1}^{*} \geq$ $-(c B) \cdot u_{2}^{*} \geq \cdots \geq-(c B) \cdot u_{p}^{*}$ where $\bar{\tau} \supseteq\{1,2, \ldots, p\}$.

Claim. Let $N^{1}:=\left\{u \in R^{m}: B^{\overline{\tau \cup\{1\}}} u \leq v^{\overline{\tau \cup\{1\}}},-(c B) \cdot u \leq-(c B) \cdot u_{1}^{*}\right\}$. Then $u_{1}^{*}$ is the unique lattice point in $N^{1}$ and all inequalities are essential.

Proof. Since $u_{1}^{*}$ lies in $R^{1}, 0=-(c B) \cdot 0 \geq-(c B) \cdot u_{1}^{*}$. However, $0>-(c B) \cdot u_{1}^{*}$ since otherwise, both $u_{1}^{*}$ and 0 would be optimal solutions to $\min \{-(c B) \cdot u: u \in$ $\left.R^{1}\right\}$ contradicting that $c$ is generic. Therefore, $N^{1}=R^{1} \cap\left\{u \in R^{m}:-(c B) \cdot u \leq\right.$ $\left.-(c B) \cdot u_{1}^{*}\right\}=\left(E^{1} \cup P_{v}^{\bar{\tau}}(0)\right) \cap\left\{u \in R^{m}:-(c B) \cdot u \leq-(c B) \cdot u_{1}^{*}\right\}=\left(E^{1} \cap\{u \in\right.$ $\left.\left.R^{m}:-(c B) \cdot u \leq-(c B) \cdot u_{1}^{*}\right\}\right) \bigcup\left(P_{v}^{\bar{\tau}}(0) \cap\left\{u \in R^{m}:-(c B) \cdot u \leq-(c B) \cdot u_{1}^{*}\right\}\right)$.

Since $c$ is generic, $u_{1}^{*}$ is the unique lattice point in the first polytope and the second polytope is free of lattice points. Hence $u_{1}^{*}$ is the unique lattice point in $N^{1}$. The relaxation of $N^{1}$ got by removing $b_{j} \cdot u \leq v_{j}$ is the polyhedron $N^{1} \cup\left(E^{j} \cap\left\{u \in R^{m}:-(c B) \cdot u \leq-(c B) \cdot u_{1}^{*}\right\}\right)$ for $j \in \bar{\tau}$ and $j \neq 1$. Either this is unbounded, in which case there is a lattice point $u$ in this relaxation such that $-(c B) \cdot u_{1}^{*} \geq-(c B) \cdot u$ or (if $j \leq p$ ), we have $-(c B) \cdot u_{1}^{*} \geq-(c B) \cdot u_{j}^{*}$ and $u_{j}^{*}$ lies in this relaxation which shows that $b_{j} \cdot u \leq v_{j}$ is essential for $N^{1}$.

Translating $N^{1}$ by $-u_{1}^{*}$ we get $P_{v^{\prime}}^{\overline{\tau \cup\{1\}}}(0):=\left\{u \in R^{m}:-(c B) \cdot u \leq 0, B^{\overline{\tau \cup\{1\}}}\right.$.

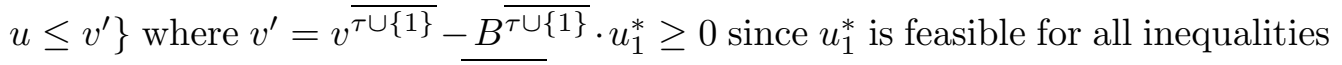
except the first one. Now $P_{v^{\prime}}^{\overline{\tau \cup\{1\}}}(0)$ contains only the origin as an integral vector and hence $\left(x^{v^{\prime}}, \tau \cup\{1\}\right)$ is a standard pair of $\operatorname{in}_{c}\left(I_{\mathcal{L}}\right)$.

Remark 3.2. Theorem 3.1 fails for general monomial ideals. The associated primes of the monomial ideal $\left\langle x^{2} y z, x y^{2} z, x y z^{2}\right\rangle \subset k[x, y, z]$ are $\langle x\rangle,\langle y\rangle,\langle z\rangle$, and $\langle x, y, z\rangle$ and each has multiplicity one. However, there is no associated prime generated by a pair of variables.

Remark 3.3. Theorem 3.1 may fail when the cost vector is not generic. We thank David Eisenbud and Sorin Popescu for pointing us to the following example. Consider the toric ideal $I_{A}$ where

$$
A=\left(\begin{array}{lllllllll}
3 & 2 & 2 & 1 & 1 & 0 & 0 & 0 & 0 \\
0 & 1 & 0 & 2 & 0 & 3 & 2 & 1 & 0 \\
0 & 0 & 1 & 0 & 2 & 0 & 1 & 2 & 3
\end{array}\right)
$$

and the cost vector $\omega=(1,1,1,1,1,1,1,1,0)$. Then $i n_{\omega}\left(I_{A}\right)=\left\langle h^{2}, g h, c h, e g, e^{2}\right.$, $c e, a e h, g^{2}-f h, c g-b h, e f-d h, c f-b g, d e-b h, b e-a h, d^{2}-b f, c d-a g, b d-$ $\left.a f, c^{2}-a e, b^{2}-a d\right\rangle$ which shows that $\omega$ is not generic. Here, the variables $a, b, c, d, e, f, g, h, i$ have been associated with the columns of $A$. The associated primes of $i n_{\omega}\left(I_{A}\right)$ are $P_{1}=\left\langle c, e, g, h, d^{2}-b f, b d-a f, b^{2}-a d\right\rangle$ of dimension three and $P_{2}=\langle a, b, c, d, e, f, g, h\rangle$ of dimension one. The embedded prime $P_{2}$ contains the minimal prime $P_{1}$. However, there is no embedded prime of dimension two for this initial ideal. 
Since the dimension of $\operatorname{in}_{c}\left(I_{\mathcal{L}}\right)$ is $n-m$, the length of a maximal chain in $\operatorname{Ass}\left(\operatorname{in}_{c}\left(I_{\mathcal{L}}\right)\right)$, which we denote as length $\left(\operatorname{Ass}\left(\operatorname{in}_{c}\left(I_{\mathcal{L}}\right)\right)\right)$, is at most $n-m$. However, when $m$ which is the codimension of $\operatorname{in}_{c}\left(I_{\mathcal{L}}\right)$ is small compared to the dimension of $\operatorname{in}_{c}\left(I_{\mathcal{L}}\right)$, length $\left(\operatorname{Ass}\left(\operatorname{in}_{c}\left(I_{\mathcal{L}}\right)\right)\right)$ has a stronger upper bound as shown below. We need the following result (Corollary 16.5a in [Sch]).

Theorem 3.4. Let $A x \leq b$ be a system of linear inequalities in $n$ variables, and let $c \in R^{n}$. If $\max \left\{c \cdot x: A x \leq b, x \in Z^{n}\right\}$ is a finite number, then max $\left\{c \cdot x: A x \leq b, x \in Z^{n}\right\}=\max \left\{c \cdot x: A^{\prime} x \leq b^{\prime}, x \in Z^{n}\right\}$ for some subsystem $A^{\prime} x \leq b^{\prime}$ of $A x \leq b$ with at most $2^{n}-1$ inequalities.

Theorem 3.5. The length of a maximal chain in $A s s\left(i n_{c}\left(I_{\mathcal{L}}\right)\right)$ is at most $\min \left(n-m, 2^{m}-(m+1)\right)$.

Proof. As we noted before we have length $\left(\operatorname{Ass}\left(\operatorname{in}_{c}\left(I_{\mathcal{L}}\right)\right)\right) \leq(n-m)$. Suppose $x^{v}$ is a standard monomial of $\operatorname{in}_{c}\left(I_{\mathcal{L}}\right)$. Then the origin is the optimal solution to the integer program (2): $\min \left\{-(c B) \cdot u: B u \leq v, u \in Z^{m}\right\}$. By Theorem 3.4 , we need at most $2^{m}-1$ inequalities to describe the same integer program. This means we can remove at least $n-\left(2^{m}-1\right)$ inequalities from $B u \leq v$ without changing the optimal solution. After removing these inequalities the corresponding polytope $P_{v}^{\bar{\tau}}(0)$ will meet the conditions in Theorem 2.5 for some $\tau \in \Delta_{c}$. Therefore $\tau$ is of size at least $n-\left(2^{m}-1\right)$. This implies that the maximal length of a chain in $\operatorname{Ass}\left(\operatorname{in}_{c}\left(I_{\mathcal{L}}\right)\right)$ is at most $(n-m)-\left(n-\left(2^{m}-1\right)\right)=2^{m}-(m+1)$.

Corollary 3.6. The dimension of an associated prime of $i n_{c}\left(I_{\mathcal{L}}\right)$ is at least $\max \left(0, n-\left(2^{m}-1\right)\right)$ and the codimension is at most $\min \left(n, 2^{m}-1\right)$.

Corollary 3.7. If $\mathcal{L}$ has rank two, then length $\left(\operatorname{Ass}\left(\operatorname{in}_{c}\left(I_{\mathcal{L}}\right)\right)\right) \leq 1$.

Proof. In this situation, $2^{m}-(m+1)=4-(4-2+1)=4-3=1$.

Remark 3.8. In $[\mathrm{HT}]$ we describe a non-Buchberger algorithm to construct $\operatorname{in}_{c}\left(I_{\mathcal{L}}\right)$ when $I_{\mathcal{L}}$ is toric. The algorithm proceeds by building $\operatorname{in}_{c}\left(I_{\mathcal{L}}\right)$ from localizations of $\operatorname{in}_{c}\left(I_{\mathcal{L}}\right)$ at its associated primes starting at the top level of $\Delta_{c}$ (i.e. at the minimal primes) and then working down the poset one level at a time. Theorems 3.1 and 3.5 provide stopping criteria and bounds for this algorithm.

We conclude this section with a family of lattice ideals of codimension $m$ for which the length of $\operatorname{Ass}\left(\operatorname{in}_{c}\left(I_{\mathcal{L}}\right)\right)$ attains the bound in Theorem 3.5. This family was used in [PS] to give lattice ideals with maximal projective dimension.

Proposition 3.9. For each $m>1$, there is a codimension $m$ lattice ideal and a cost vector $c \in R^{n}$ where $n=2^{m}-1$ such that length $\left(A s s\left(i_{c}\left(I_{\mathcal{L}}\right)\right)\right)=2^{m}-$ $(m+1)$. 
Proof. Given $m>1$, let $B=\left(b_{i j}\right) \in Z^{\left(2^{m}-1\right) \times m}$ be the matrix whose rows are all possible vectors in $R^{m}$ that use 1 and -1 as entries except $v=$ $(-1,-1, \ldots,-1)$. The lattice generated by the columns of $B$ has rank $m$, and therefore we can find a cost vector $c \in R^{n}$ (where $n=2^{m}-1$ ) such that $-c B=v$. For each row $b_{i}$ we set $r_{i}:=\left|\left\{b_{i j}: b_{i j}=1\right\}\right|$, and let $r$ be the vector of all $r_{i}$ 's. By construction, the polytope $P:=\left\{u \in R^{m}: B u \leq r,-(c B) \cdot u \leq 0\right\}$ has no lattice points in its interior and each of its $2^{m}$ facets has exactly one vertex of the unit cube in $R^{m}$ in its relative interior. If we let $w_{i}=r_{i}-1$, then the polytope $\left\{u \in R^{m}: B u \leq w,-(c B) \cdot u \leq 0\right\}$ is $P_{w}^{[n]}(0)$. The origin is the only lattice point in $P_{w}^{[n]}(0)$ and all the inequalities are essential. By Theorem 2.5, $\left(x^{w}, \emptyset\right)$ is a standard pair of $\operatorname{in}_{c}\left(I_{\mathcal{L}}\right)$. Since a maximal face of $\Delta_{c}$ is $\left(2^{m}-1-m\right)$ dimensional, Theorem 3.1 implies that length $\left(\operatorname{Ass}\left(\operatorname{in}_{c}\left(I_{\mathcal{L}}\right)\right)\right)=2^{m}-1-m$.

\section{Irreducible primary decomposition}

Definition 4.1. A polyhedron is maximal lattice point free if and only if it contains no interior lattice points but every facet of it contains an interior lattice point.

In Section 2 we saw that a standard pair $\left(x^{v}, \tau\right)$ of $\operatorname{in}_{c}\left(I_{\mathcal{L}}\right)$ gives the polytope $P_{v}^{\bar{\tau}}(0)=\left\{u \in R^{m}: B^{\bar{\tau}} u \leq v^{\bar{\tau}},(-c B) \cdot u \leq 0\right\}$ in which the origin is the only lattice point and all inequalities are essential. Suppose we now increase the coordinates of $v^{\bar{\tau}}$ by integral amounts until $P_{v}^{\bar{\tau}}(0)$ becomes maximal lattice point free. Then $P_{v}^{\bar{\tau}}(0)$ is a full dimensional polytope in which every row of $B^{\bar{\tau}} u \leq v^{\bar{\tau}}$ gives rise to a facet of $P_{v}^{\bar{\tau}}(0)$.

Theorem 4.2. The ideal $i n_{c}\left(I_{\mathcal{L}}\right)$ has the irreducible primary decomposition

$$
i n_{c}\left(I_{\mathcal{L}}\right)=\bigcap_{P_{q}^{\bar{\tau}}(0)}\left(x_{i}^{q_{i}}: i \in \bar{\tau}\right)
$$

where the intersection is over all maximal lattice point free polytopes of the form $P_{q}^{\bar{\tau}}(0)$.

For an arbitrary monomial ideal $M$, let $M_{\tau}$ denote its localization at the associated prime $p_{\tau}$. We identify $M_{\tau}$ with its projection in $k\left[x_{\bar{\tau}}\right]:=k\left[x_{i}: i \in \bar{\tau}\right]$ under the map $\pi_{\tau}: k\left[x_{1}, \ldots, x_{n}\right] \rightarrow k\left[x_{\bar{\tau}}\right]$ such that $x_{i} \mapsto 1$ if $i \in \tau$ and $x_{i} \mapsto x_{i}$ otherwise. Under this projection, the standard pairs of $M$ of the form $\left(x^{v}, \tau\right)$ are in bijection with the standard pairs of $M_{\tau}$ of the form $\left(x^{v}, \emptyset\right)$. For a set $J \subseteq \bar{\tau}$, let $e_{J}$ denote the vector in $N^{|\bar{\tau}|}$ with one in the positions indexed by $J$ and zero otherwise. Let $q \in N^{|\bar{\tau}|}$ be such that $q:=p+e_{\bar{\tau}}$ where $x^{p}$ is a socle element modulo $M_{\tau}$. i.e., $x^{p} x_{i} \in M_{\tau}$ for all $i \notin \tau$. ( $M_{\tau}$ has finitely many socle elements and each such $p$ gives a standard pair $\left(x^{p}, \emptyset\right)$ of $M_{\tau}$.) We now recall the following result from $[\mathrm{STV}]$. 
Lemma 4.3. [STV] $A$ monomial ideal $M$ admits the irreducible primary decomposition

$$
M=\bigcap\left(x_{i}^{q_{i}}: i \in \bar{\tau}\right)
$$

where the intersection is over all $\tau \subset[n]$ and all $q \in N^{|\bar{\tau}|}$ such that $q=p+e_{\bar{\tau}}$ and $x^{p}$ is a socle element modulo the localization of $M$ at the associated prime $p_{\tau}$.

Proof. A monomial $x^{m}$ does not lie in $M$ if and only if there is a standard pair $\left(x^{v}, \tau\right)$ of $M$ such that $x^{m}=x^{v} \cdot x_{\tau}^{t}$ for some $t \in N^{|\tau|}$. Let $x^{p}$ be a socle element of $M_{\tau}$ such that $v \leq p$. Then $x^{m}=x^{v} \cdot x_{\tau}^{t}$ if and only if $x^{m} \notin\left(x_{i}^{p_{i}+1}: i \in \bar{\tau}\right)$.

In order to prove Theorem 4.2 we establish a bijection between the socle elements of $\operatorname{in}_{c}\left(I_{\mathcal{L}}\right)_{\tau}$ and the maximal lattice point free polytopes of the form $P_{v}^{\bar{\tau}}(0)$. We assume that $p_{\tau} \in \operatorname{Ass}\left(\operatorname{in}_{c}\left(I_{\mathcal{L}}\right)\right)$.

Lemma 4.4. Let $x^{p}$ be a socle element of $i n_{c}\left(I_{\mathcal{L}}\right)_{\tau}$. Then, for $J \subseteq \bar{\tau}$, the polytope $P_{p+e_{J}}^{\bar{\tau}}(0)$ has no lattice points in its relative interior, but it contains lattice points on all of its facets indexed by $J$.

Proof. The polytope $P_{p+e_{J}}^{\bar{\tau}}(0)$ is obtained by relaxing each constraint $b_{i} \cdot x \leq p_{i}$ in $P_{p}^{\bar{\tau}}(0)$ to $b_{i} \cdot x \leq p_{i}+1$ for $i \in J$. Suppose the non-zero lattice point $z$ is in the relative interior of $P_{p+e_{J}}^{\bar{\tau}}(0)$. This implies that $b_{i} \cdot z<p_{i}+1$ for all $i \in J$, which in turn implies that $b_{i} \cdot z \leq p_{i}$ for all $i \in J$. However, this contradicts that $P_{p}^{\bar{\tau}}(0)$ has no non-zero lattice points. Since the monomial $x^{p} \cdot x_{i} \in \operatorname{in}_{c}\left(I_{\mathcal{L}}\right)_{\tau}$ for each $i \in J$, the polytope $P_{p+e_{i}}^{\bar{\tau}}(0)$ contains lattice points that satisfy $b_{i} \cdot x=p_{i}+1$. This shows that $P_{p+e_{J}}^{\bar{\tau}}(0)$ contains lattice points on all of its facets indexed by $J$.

Proposition 4.5. The polytope $P_{q}^{\bar{\tau}}(0)$ where $q \in N^{|\bar{\tau}|}$ has full support is maximal lattice point free if and only if $q=p+e_{\bar{\tau}}$ where $x^{p}$ is a socle element of $i n_{c}\left(I_{\mathcal{L}}\right)_{\tau}$

Proof. Suppose $q=p+e_{\bar{\tau}}$ where $x^{p}$ is a socle element of $\operatorname{in}_{c}\left(I_{\mathcal{L}}\right)_{\tau}$. Since $x^{q} \in \operatorname{in}_{c}\left(I_{\mathcal{L}}\right)_{\tau}, P_{q}^{\bar{\tau}}(0)$ has non-zero lattice points in it but by the above lemma, all of these points lie on the boundary. Since $q_{i}>0$ for all $i \in \bar{\tau}$, no inequality in $B^{\bar{\tau}} x \leq q$ is binding at the origin which implies that the origin lies in the interior of the facet defined by the cost vector. Suppose for some $i \in \bar{\tau}$, the facet $b_{i} \cdot x=p_{i}+1$ of $P_{q}^{\bar{\tau}}(0)$ has no lattice point in its interior. Then by tightening all of the inequalities $b_{j} \cdot x \leq p_{j}+1$ back to $b_{j} \cdot x \leq p_{j}$ for all $j \neq i$, you get a polytope with no nonzero lattice points. However, this polytope is $P_{p+e_{i}}^{\bar{\tau}}(0)$ which does contain nonzero lattice points since $x^{p} \cdot x_{i} \in \operatorname{in}_{c}\left(I_{\mathcal{L}}\right)_{\tau}$.

Conversely, suppose $P_{q}^{\bar{\tau}}(0)$ is maximal lattice point free. Notice first that the origin can be the only lattice point in the interior of the facet defined by the cost vector. Else, after tightening each of the inequalities $b_{i} \cdot x \leq q_{i}$ to $b_{i} \cdot x \leq q_{i}-1$ we get an integer program with two optimal solutions which contradicts that 
$c$ is generic. Since $P_{q}^{\bar{\tau}}(0)$ is maximal lattice point free, the body $P_{q-e_{\bar{\tau}}}^{\bar{\tau}}(0)$ has no lattice points other than the origin. Therefore, $x^{p}=x^{q-e_{\bar{\tau}}}$ is a standard monomial of $\operatorname{in}_{c}\left(I_{\mathcal{L}}\right)_{\tau}$. Also, since there are lattice points in the interior of every facet, every intermediate body got by tightening a subset of the inequalities in $B^{\bar{\tau}} x \leq q$ contains non-zero lattice points. This implies that $x^{p}$ is a socle element of $\operatorname{in}_{c}\left(I_{\mathcal{L}}\right)_{\tau}$.

Proof of Theorem 4.2: The result follows from Lemma 4.3 and Proposition 4.5.

\section{References}

[BSS] I. Barany, H.E. Scarf and D. Shallcross, The topological structure of maximal lattice point free convex bodies: the general case, Integer Programming and Combinatorial Optimization, Lecture Notes in Computer Science 920, Springer-Verlag, Berlin, 1995, pp. 244-251.

[BM] D. Bayer and D. Mumford, What can be computed in algebraic geometry?, in Computational Algebraic Geometry and Commutative Algebra, ( D. Eisenbud and L. Robbiano, eds.), Symposia Mathematica XXXIV, Cambridge University Press, Cambridge, 1993, pp. $1-48$.

[BFS] L.J. Billera, P. Filliman and B. Sturmfels, Constructions and complexity of secondary polytopes, Adv. in Math. 83 (1990), 155-179.

[CT] P. Conti and C. Traverso, Buchberger Algorithm and Integer Programming, Applied algebra, algebraic algorithms and error-correcting codes (New Orleans, LA, 1991), 130-139, Lecture Notes in Comput. Sci., 539, Springer, Berlin, 1991.

[Ful] W. Fulton, Introduction to Toric Varieties, Annals of Mathematics Studies, 131, The William H. Roever Lectures in Geometry. Princeton University Press, Princeton, NJ, 1993.

[HT] S. Hoşten and R.R. Thomas, Standard pairs and group relaxations in integer programming, to appear in J. Pure Appl. Algebra .

[Lov] L. Lovász, Geometry of numbers and integer programming, in Mathematical Programming: Recent Developments and Applications, ( M. Iri and K. Tanebe, eds.), Kluwer Academic Press, 1989, pp. 177-210.

[PS] I. Peeva and B. Sturmfels, Syzygies of codimension 2 lattice ideals, Math. Z. 229 (1998), 163-194.

[Sch] A. Schrijver, Theory of Linear and Integer Programming, Wiley-Interscience Series in Discrete Mathematics and Optimization, John Wiley \& Sons, Ltd., New York, 1986.

[Stu] B. Sturmfels, Gröbner Bases and Convex Polytopes, Amer. Math. Soc., Providence, RI, 1995.

[STV] B. Sturmfels, N. Trung and W. Vogel, Bounds on projective schemes, Math. Ann. 302 (1995), 417-432.

[SWZ] B. Sturmfels, R. Weismantel and G. Ziegler, Gröbner bases of lattices, corner polyhedra and integer programming, Beiträge Algebra Geom. 36 (1995), 281-298.

Mathematical Sciences Department, George Mason University, Fairfax, VA 22030

E-mail address: shosten@gmu.edu

Department of Mathematics, Texas A\&M University, College Station, TX 77843

E-mail address: rekha@math.tamu.edu 